\title{
Determination of norflurazon concentration in wheat leaves using a modified QuEChERS method*
}

\author{
Kamil F. Trzebuniak and Beata Mysliwa-Kurdziel ${ }^{\bowtie}$ \\ Department of Plant Physiology and Biochemistry, Faculty of Biochemistry, Biophysics and Biotechnology, Jagiellonian University, \\ Kraków, Poland
}

The aim of this analytical study was to develop and validate an easy-to-use method for measuring the actual level of norflurazon that accumulates in leaves. We amended the QuEChERS method, i.e. Quick, Easy, Cheap, Effective, Rugged, and Safe, which is widely used for pesticide and herbicide analysis in food, and usually combined with HPLC-MS detection. We adapted this method for the detection of norflurazon in leaves or leaf fragments and proposed a useful modification using of HPLC-UV detection. Reproducible retention times of $3.11 \pm 0.04 \mathrm{~min}$, precision ( $\mathrm{RSD}<8.0 \%$ ), $\mathrm{LOQ}=315 \mathrm{ng} \cdot \mathrm{mL}^{-1}$ and linearity $\left(R^{2}=0.99874\right)$ were achieved.

Key words: norflurazon, QuEChERS, Wheat, Triticum aestivum, HPLCUV

Received: 22 March, 2017; revised: 28 April, 2017; accepted: 01 August, 2017; available on-line: 19 August, 2017

$\triangle$ e-mail: b.mysliwa-kurdziel@uj.edu.pl

* This work was, in part, presented at the XLIV Winter School of the Faculty of Biochemistry, Biophysics and Biotechnology of the Jagiellonian University in Cracow "No stress - no life", Zakopane, Poland, 14-18th February 2017.

Abbreviations: ACN, acetonitrile; DAD, diode-array detector; HPLC, high performance liquid chromatography; LOD, limit of detection; LOQ, limit of quantification; MS, mass spectrometry; NF, norflurazon; QuEChERS, quick, easy, cheap, effective, rugged, and safe; RSD, relative standard deviation; UV-Vis, ultraviolet-visible spectroscopy

\section{INTRODUCTION}

Norflurazon (4-Chloro-5-(methylamino)-2-( $\alpha, \alpha, \alpha$-trifluoro- $m$-tolyl)-3(2H)-pyridazinone; NF) (Fig. 1) is a bleaching herbicide used to treat weeds. It works by inhibiting phytoene desaturase (EC 1.3.99.30) (Kümmel \& Grimme, 1975; Breitenbach et al., 2001) and leads to the arrest of carotenoid synthesis (Sandmann et al., 1989). Chlorophyll excitation into an unquenched triplet state in the absence of carotenoids induces the formation of reactive oxygen species, photooxidation processes, chloroplast damage, and finally results in plant photobleaching.

NF is also useful in plant science to study the mechanisms of oxidative stress as well as their influence on photosynthesis and chloroplast biogenesis, especially ret-<smiles>CNc1cnn(-c2cccc(C(F)(F)F)c2)c(=O)c1Cl</smiles>

Figure 1. Structure of norflurazon (NF): 4-Chloro-5(methylamino)-2-(a,a,a-trifluoro- $m$-tolyl)-3(2H)-pyridazinone rograde chloroplast-nucleus signalling (Terry \& Smith, 2013). A reduction in the expression of many nuclear genes has already been demonstrated in plants treated with this herbicide (Oelmüller et al., 1986; Strand et al., 2003; Moulin et al., 2008). However, taking into account that different ways of applying herbicides are currently used in different studies e.g. spraying (Van Aken et al., 2016) and watering (Burgess et al., 2016), explaining the NF effect and comparing results of various studies requires the actual concentration of $\mathrm{NF}$ in plant tissue to be determined. In the above mentioned papers the authors use a different concentration of $\mathrm{NF}$, between $1.52-15.18 \mu \mathrm{g} \cdot \mathrm{mL}^{-1}$ (i.e. between 5 and $50 \mu \mathrm{mol} \cdot \mathrm{L}^{-1}$ ).

The QuEChERS method (quick, easy, cheap, effective, rugged, and safe) was developed in 2003 (Anastassiades et al., 2003) and found widespread application in food analysis. The method was standardised in Europe (EN 15662:2008) and in the United States (AOAC International, 2007). The most important step in the QuEChERS method is dispersive solid phase extraction using secondary-primary amine sorbents. There are multiple methods derived from the standard QuEChERS method, which focus on unusual or challenging matrices - Gil Garcia and coworkers (2017) write about over 100 variations. It has also been used in order to detect up to 450 pesticides simultaneously (Pang et al., 2006). Currently, the gold standard for pesticide and herbicide detection in food are methods based on gas or high performance liquid chromatography coupled with (tandem) mass spectrometry (HPLC-MS). This is also the detection method described in the QuEChERS procedure. Although the mass spectrometer is a very sensitive and universal detector, it is not affordable for many laboratories. When only a single (or just a few) analyte concentration needs to be determined, while keeping the chromatographic procedure short and simple, there is the possibility of using a different detector.

The aim of this study was to develop a simple QuEChERS-based method, with a diode-array-ultraviolet detector (DAD-UV), for measurements of NF concentration in juvenile wheat leaves and leaf fragments. Wheat, which is the second most cultivated crop in the world (document FAO UN, 2017), is often used for studying deetiolation due to the ease of cultivating it and its fast growth. In addition to this, being monocot plants, wheat seedlings accumulate plastids that show a developmental gradient from the pregranal plastids at the leaf base to the eldering etioplasts at the leaf tip (Solymosi \& Aronsson, 2013).

\section{MATERIALS AND METHODS}

Chemicals and reagents. Norflurazon (NF) analytical standards, QuEChERS sorbents and tubes (PSA/ 
ENVI-Carb Tube 2, suitable for EN 15662:2008 per BS) were purchased from Sigma-Aldrich. HPLC-grade acetonitrile (ACN) and water, purchased from Honeywell, were used unless indicated otherwise. Analytical grade reagents purchased from Chempur or Sigma-Aldrich were used unless indicated otherwise.

Wheat cultivation. Wheat seedlings (Triticum aestivum L.) were hydroponically cultivated on Hoagland salt $\left(0.16 \mathrm{~g} \cdot \mathrm{L}^{-1}\right.$; Sigma-Aldrich) at $295 \mathrm{~K}$. They were grown in darkness for 7 days and for the following 24 hours they were treated with white light of $100 \mu \mathrm{mol}$ of photons $\cdot \mathrm{m}^{-2} \cdot \mathrm{s}^{-1}$. For NF-treated samples, NF was added to the cultivation medium at the beginning of the cultivation period at a concentration of $6.1 \mu \mathrm{g} \cdot \mathrm{mL}^{-1}$. For NF-spraying experiments the culture was sprayed daily with a $6.1 \mu \mathrm{g} \cdot \mathrm{mL}^{-1} \mathrm{NF}$ solution using a spray bottle. Care was taken to minimise spraying the cultivation medium.

The leaves were harvested under dim green light in order to prevent photoxidation of the pigments (Holden, $1965)$ and were cut into pieces of about $1 \mathrm{~cm}$. The pieces of leaves were analysed immediately after harvesting or stored at $193 \mathrm{~K}$ in the absence of light.

Sample preparation. The samples were prepared using a modified QuEChERS method (European Committee for Stantarization, 2008). A pooled portion of $7.0 \pm 0.1 \mathrm{~g}$ of leaf fragments was collected and handground with liquid nitrogen $(77 \mathrm{~K}$ ) using a pestle and mortar. The homogenate was quantitatively transferred with ACN into a $50 \mathrm{~mL}$ plastic tube, using $10 \mathrm{~mL}$ of ACN. The tube was shaken vigorously for 1 minute using a lab vortex. A buffer-salt mixture (4.0 g anhydrous magnesium sulphate(VI), $1.0 \mathrm{~g}$ sodium chloride, $1.0 \mathrm{~g}$ trisodium citrate dihydrate, $0.5 \mathrm{~g}$ disodium hydrogencitrate sesquihydrate) was added to the suspensions and the tubes were shaken again for 1 minute. The samples were then centrifuged for 5 minutes at $3100 \times g$, at $277 \mathrm{~K}$. Then the sample was placed in a freezer overnight to precipitate the waxes and sugars. After that time an aliquot of $6 \mathrm{~mL}$ of the ACN phase was transferred to dispersive-SPE QuEChERS tubes containing an amino-sorbent, graphitized non-porous carbon and magnesium sulphate $(V I)$, and shaken vigorously for 2 minutes. The samples were then centrifuged for 5 minutes at $3100 \times g$ at $277 \mathrm{~K}$. Then $5 \mathrm{~mL}$ of the ACN phase was transferred to a new tube, evaporated until dry and dissolved in $500 \mu \mathrm{L}$ of the mobile phase $(60 \%$ ACN in HPLC-grade water). Finally the samples were filtrated using centrifugal filtering tubes (Costar Spin-X $0.22 \mu \mathrm{m}$ ) for $90 \mathrm{sec}-$ onds at $11000 \times g$.

Chromatography and norflurazon detection. The samples were separated chromatographically using a JASCO PU-2089 Plus HPLC system with a JASCO MD-2015plus DAD-UV-VIS detector. The data were obtained using JASCO ChromPass 1.8.6.1 software. $100 \mu \mathrm{L}$ of the sample was injected with a Rheodyne Manual Sample Injector (7725i). They were separated with a C18 column (Teknokroma TRACER EXCEL 120 ODSA $3 \mu \mathrm{m} 15 \times 0.4 \mathrm{~cm})$. An isocratic elution with $60 \%$ $\mathrm{ACN}$ at $1.0 \mathrm{~mL} \cdot \mathrm{min}^{-1}$ was used. The total run time was 5 minutes. The chromatographic system was equilibrated for at least 20 minutes with the mobile phase and a flowrate of $1.0 \mathrm{~mL} \cdot \mathrm{min}^{-1}$ before the first run of the day. NF was detected at $\lambda=296 \mathrm{~nm}$. Additionally spectra between 200 and $400 \mathrm{~nm}$ were collected in order to confirm the NF identification by UV spectrum. The spectra were collected every $400 \mathrm{~ms}$ with a resolution of $3 \mathrm{~nm}$.

Method validation. The quantitative procedure and the method validation were performed by assessing the recovery and clean-up, precision, carry-over, LOD and LOQ and linearity. SANCO guidelines on analytical quality control and validation procedures (document SANTE/11945/2015) were applied unless indicated otherwise.

For the recovery measurements, blank samples (10 $\mathrm{mL} \mathrm{ACN}$ ) were spiked with $\mathrm{NF}$ to a concentration of $0.5 \mu \mathrm{g} \cdot \mathrm{mL}^{-1}$. The samples were evaporated until dry using a rotavapor, dissolved in the mobile phase, filtered and analysed by HPLC-UV. The NF concentration was compared with the blank samples $(10 \mathrm{~mL}$ ACN) spiked with the same amount of NF and prepared as described in the "sample preparation" section.

The method was checked for carry-over effects. First the chromatogram of a control sample consisting of a leaf extract was recorded. Then the NF spiked sample was injected. The NF concentration chosen was twice as high as in the most abundant NF-treated leaf sample. Finally a blank sample $(60 \%$ ACN) was injected. All the samples were injected directly one after the other without flushing the column.

The limit of detection (LOD) was calculated by analysing the control samples (leaves not treated with NF). The sample with the highest background between 3.00 and 3.20 minutes at $\lambda=296 \mathrm{~nm}$ was selected. The LOD was calculated as 3 times the height of this background. The limit of Quantification (LOQ) was assumed to be 9 times the height of the background, because at this concentration the precision appeared satisfactory.

Linearity was analysed using NF-spiked samples containing $5 \mu \mathrm{g} \cdot \mathrm{mL}^{-1} \mathrm{NF}$, diluted 2 fold until the calculated LOD was achieved.

Precision was calculated using data gathered from linearity experiments.

Statistical analysis. The retention time of NF was calculated by taking the mean of $n=37$ curves, i.e. the chromatograms recorded for all the spiked samples used in the calibration curve, those of carry-over experiments and those of all plant extracts, where NF was added to the medium or used to spray the seedlings. In all the cases the NF peak was determined by the UV spectrum. The error of retention time is presented as the calculated standard deviation (S.D.).

The recovery was calculated in triplicate, and the respective value is presented as the mean value \pm S.D.

All linearity experiments were performed in triplicate. The peak amplitude was determined automatically using the software provided by the manufacturer of the HPLC-UV system (JASCO ChromPass Chromatography Data System v 1.8.6.1). Results for different concentrations are represented as the mean value \pm S.D. Linear regression and slope (method sensitivity) were calculated using OriginPro 2016 b9.3.2.303 software. The results obtained for six NF concentrations were taken into account for the regression calculation.

The method precision was determined during the linearity measurements. The precision is represented as relative standard deviation (RSD) calculated separately for each concentration.

\section{RESULTS AND DISCUSSION}

The presence of NF was confirmed by comparing the spectra recorded, with the spectra of the NF standard measured using a Jasco V-650 spectrophotometer (Fig. 2). The absorption spectrum of NF is complex and has tree maxima at 209, 238 and $296 \mathrm{~nm}$. The spectra shown in Fig. 2 were virtually identical; the $2 \mathrm{~nm}$ dif- 

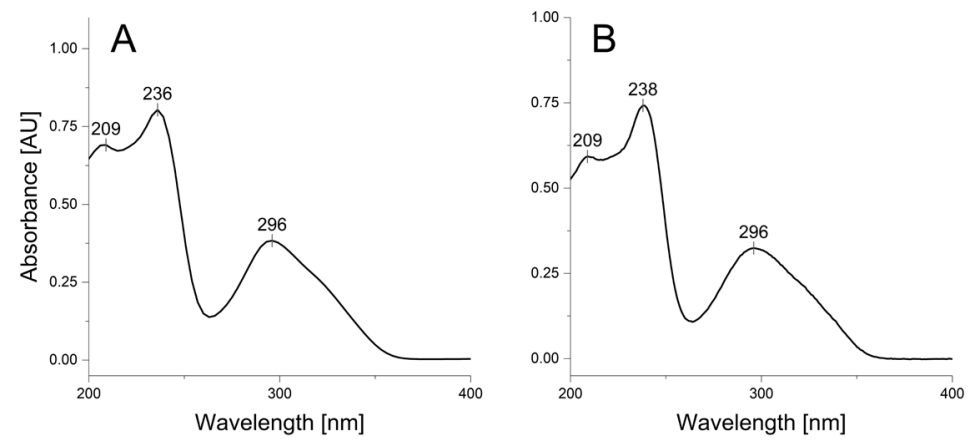

Figure 2. Absorption spectra of NF

(A) the spectrum for the recorded chromatographic peak at $3.11 \mathrm{~min}$; (B) the NF standard dissolved in $60 \%$ ACN. Visible peaks are annotated with the wavelength of their local maximum.
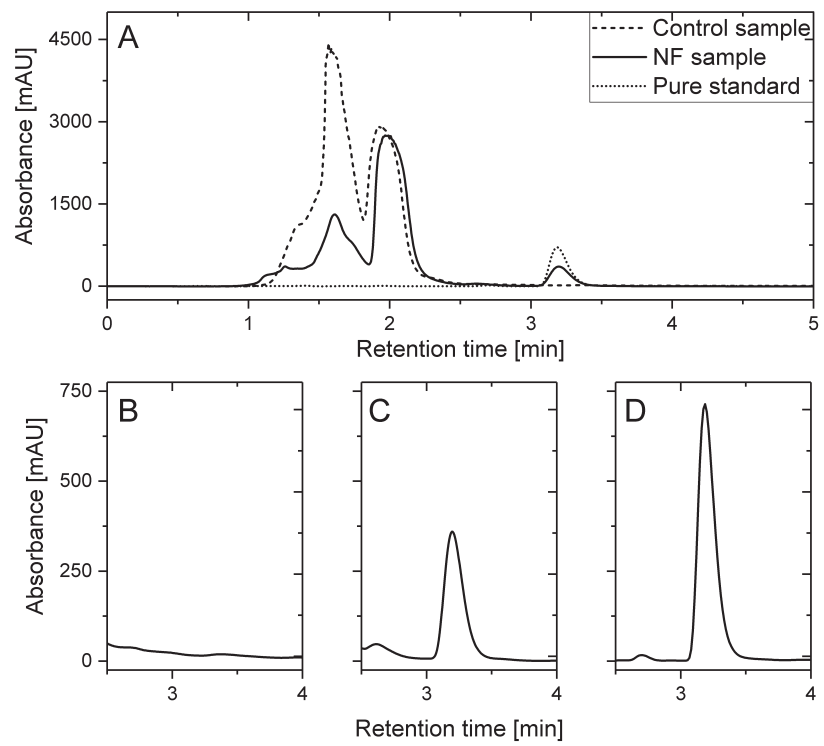

Figure 3. Representative chromatograms (A) of a control sample (dashed line), a sample from NF-treated plants (solid line) and a pure NF standard dissolved in the mobile phase (dotted line), recorded at $\lambda=296 \mathrm{~nm}$. The area between 2.5 and 4.0 minutes enlarged 6.5-fold for the control sample (B), the NF-treated sample (C) and the pure NF standard dissolved in the mobile phase (D).

ference in the position of the highest peak is within the detector's uncertainty range. A wavelength of $296 \mathrm{~nm}$ was selected for NF detection due to a low and stable baseline. A wavelength of $238 \mathrm{~nm}$ was also tested but found unsatisfactory due to a greater baseline drift. Furthermore, a NF-treated leaf sample was analysed and spiked with additional NF. This resulted in the peak area and amplitude increasing, without a change in the retention time.

A simple, isocratic elution was used, allowing for a short total run time of 5 minutes (Fig. 3) without the need for reequilibration. We achieved a retention time of $3.11 \pm 0.04 \mathrm{~min}(\mathrm{n}=37)$ and resolution above 2 (calculated using the base-width 4 sigma method (Neue, 1997). The dead time was about 1 minute, followed by a very high matrix peak between 1.25 and 2.00 minutes. The height of this peak varied between samples. Around 2.55 min there is a small peak in the NF-treated samples. It is possible that this is from some NF metabolites but we were unable to verify this unambiguously. Additionally, more elaborated elution programs were tested, but resulted in worse resolutions or longer analysis times due to the need for column reequilibration (not shown).
Recovery was calculated in triplicate for an NF concentration of $0.5 \mu \mathrm{g} \cdot \mathrm{mL}^{-1}$. The result was the recovery of $79.8 \pm 1.5 \%$ of the analyte.

Carry-over effects may result in false positive samples when a blank sample is analysed directly after a sample with a high concentration of the analyte. In order to check for those effects three chromatograms were recorded as follows: a control sample (Fig. 4B), a high NF concentration spiked sample (Fig. 4C), and a blank sample (Fig. 4D). The concentration of NF in the spiked sample was chosen so that the recorded signal was as close as possible to the highest value that can be recorded using our detector setup and is twice as large as the greatest signal recorded from NF-treated leaves. The concentration of $\mathrm{NF}$ measured in the final sample was below the LOD, and virtually no rise to the baseline was visible (Fig. 4A).

In order to calculate the LOD, six control samples consisting of non-NF-treated leaf extracts were analysed. The highest baseline between 3.00 and 3.20 minutes at a wavelength of $296 \mathrm{~nm}$ was selected for further calculations. LOD was calculated as three times the value of the baseline and was equal to $120 \mathrm{ng} \cdot \mathrm{mL}^{-1}$. LOQ was calculated as nine times the 

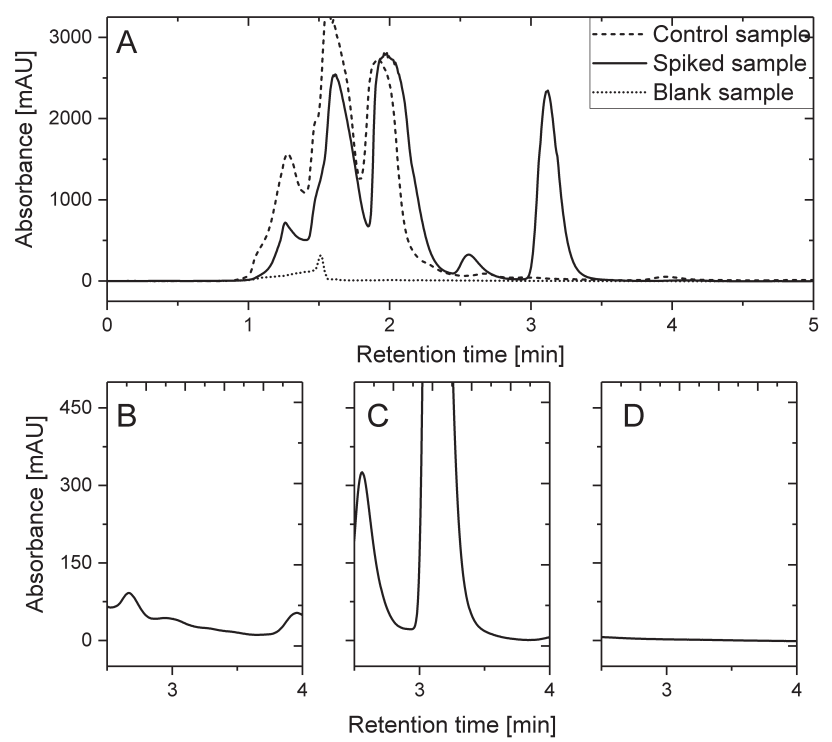

Figure 4. The carry-over experiment.

(A) representative chromatograms of a control sample (i.e. an extract from control leaves that were not treated with NF; dashed line), a NF-spiked sample (solid line) and a blank sample (ACN, dotted line). Chromatograms were recorded at $\lambda=296 \mathrm{~nm}$ directly one after the other without washing or reequilibration between them. The area between 2.5 and 4.0 minutes enlarged 6 -fold for the control sample (B), the NF-spiked sample (C) and the blank sample (D).

baseline and equalled $315 \mathrm{ng} \cdot \mathrm{mL}^{-1}$. These values are much smaller than those found in NF-treated plants (usually between $700 \mathrm{ng} \cdot \mathrm{mL}^{-1}$ and $1.50 \mu \mathrm{g} \cdot \mathrm{mL}^{-1}$ ) which suffer a visible bleaching effect.

Linearity was measured in triplicate starting from a $\mathrm{NF}$-spiked sample containing $5 \mu \mathrm{g} \cdot \mathrm{mL}^{-1} \mathrm{NF}$, diluted 2 fold until a concentration below the LOD calculated was achieved (ca. $84 \mathrm{ng} \cdot \mathrm{mL}^{-1}$ ). The sensitivity calculated was $230.5 \pm 3.7 \mathrm{mAU} \cdot \mu \mathrm{g} \cdot \mathrm{mL}^{-1}$. A very good fit was found through the entire range measured $R^{2}=0.99874$ $\left(\mathrm{A}=230.5 \cdot C_{\mathrm{m}}-2.5\right.$; where $\mathrm{A}-$ absorbance in $\mathrm{mAU}, C_{\mathrm{m}}-$ mass concentration in $\mu \mathrm{g} \cdot \mathrm{mL}^{-1}$ ) (Fig. 5).

The measurements for the linear range were used to assess the precision of the methods. The relative standard deviation (RSD) was lower than $8.0 \%$ for all measurements (ca. 6\% for most measurements).

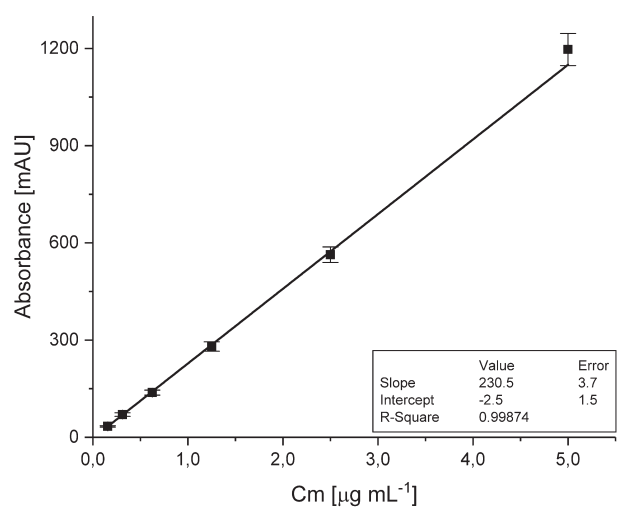

Figure 5. Calibration curve prepared by triplicate measurements of spiked samples at NF levels of: $5.00 \mu \mathrm{g} \times \mathrm{mL}^{-1} ; 2.50 \mu \mathrm{g} \times \mathrm{mL}^{-1}$; $1.25 \mu \mathrm{g} \times \mathrm{mL}^{-1} ; 625 \mathrm{ng} \times \mathrm{mL}^{-1} ; 313 \mathrm{ng} \times \mathrm{mL}^{-1}$; and $156 \mathrm{ng} \times \mathrm{mL}^{-1}$. The corresponding RSDs were as follows: $4.1 \%, 4.2 \%, 5.2 \%, 5.6 \%$, $7.8 \%, 6.4 \%$
Plants treated with norflurazon are easily distinguishable from control plants due to the bleaching effect of herbicides. As demonstrated in Fig. 6 the control plants underwent greening as expected (Fig. 6A). In contrast, the NF-treated plants were mostly white or light yellow (Fig. 6B). No observable difference in leaf size was visible under our cultivation conditions.

In the literature different methods of applying $\mathrm{NF}$ can be found e.g. supplementation of the growth medium or spraying different parts of the plant. The concentration of NF in the growth medium or in the solution used to spray the plants is specified, but comparing the results is challenging since there is no information about the actual NF concentration in the plant tissue. We have demonstrated (Fig. 7) that the modified QuEChERS method can be helpful in this application. For the control sample the measured signal was $3.5 \mathrm{mAU}$ which corresponds to $26 \mathrm{ng} \cdot \mathrm{mL}^{-1}$ and is far below the LOD and can be explained by random noise. The NF-sprayed sample generated a signal of $80.2 \mathrm{mAU}$, which corresponds to a NF concentration of $359 \pm 6 \mathrm{ng} \cdot \mathrm{mL}^{-1}$. The sample where NF was added to the growth medium provided a signal of $181.9 \mathrm{mAU}$, which corresponds to $800 \pm 13 \mathrm{ng} \cdot \mathrm{mL}^{-1}$. Further calculations per gram of fresh leaf mass provided a NF amount of $512 \pm 8 \mathrm{ng}$ in NF-sprayed samples and of $1143 \pm 19 \mathrm{ng}$ in samples supplied with $\mathrm{NF}$ in the growth medium.
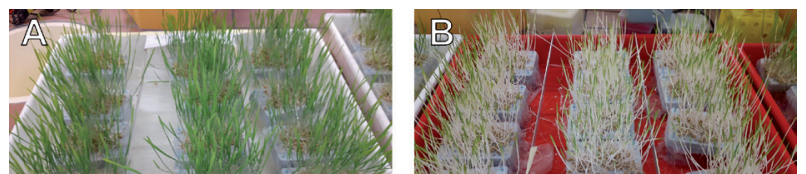

Figure 6. Photographs of wheat after 7 days of hydrophonic cul-

tivation.
(A) control plants (not treated with NF) (B) plants cultivated with the NF added to the medium to a final concentration of 6.1 $\mathrm{mg} \times \mathrm{L}^{-1}$ 

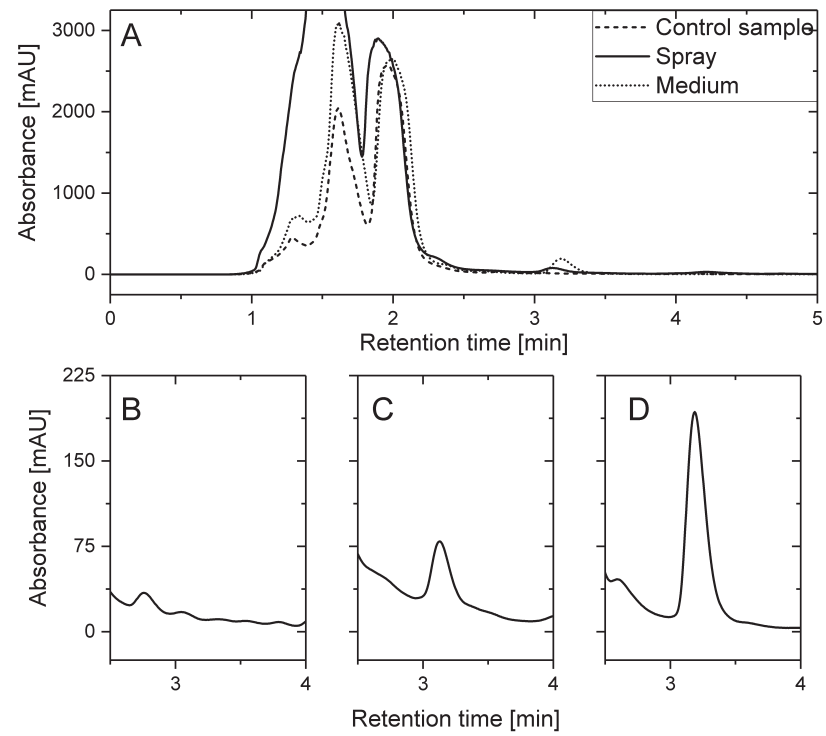

Figure 7. Comparison of different methods of applying NF.

(A) representative chromatograms of a control sample (not treated with NF; dashed line), a sample sprayed daily with a $6.1 \mathrm{mg} \mathrm{L}^{-1} \mathrm{NF}$ solution (solid line) and a sample cultivated with NF added to the cultivation medium to a concentration of $6.1 \mathrm{mg} \mathrm{L}^{-1}$ (dotted line), recorded at $\lambda=296 \mathrm{~nm}$. The area between 2.5 and 4.0 minutes enlarged 14-fold for the control sample (B), the sample from NF-sprayed plants (C) and the sample from plants cultivated with NF addition to the medium (D).

\section{CONCLUSION}

The QuEChERS with UV-Vis detection developed and validated in this work is useful for determining the amount of NF that accumulates in a plant tissue, seedlings, leaves or leaf fragments. It is useful for comparing the distribution of NF among different parts of a plant or among leaf fragments. NF detection is not disturbed by the presence of chlorophylls in samples. Therefore this method allows us to study the influence of NF on photosynthetic processes.

\section{Declaration of conflicting interest}

The authors declare that there is no conflict of interest.

\section{Acknowledgements}

We would like to thank Agata Zubrycka, Natalia Duraczyńska, Dominika Zuk and Stanisław Listwan for their help with the sample collection.

The Faculty of Biochemistry, Biophysics and Biotechnology of the Jagiellonian University is a partner of the Leading National Research Center (KNOW) supported by the Ministry of Science and Higher Education.

This work was financially supported by grant UMO2013/10/E/NZ3/00748 from the Polish National Science Centre (NCN).

\section{REFERENCES}

Anastassiades M, Lehotay SJ, Stajnbaher D, Schenck FJ (2003) Fast and easy multiresidue method employing acetonitrile extraction/partitioning and "dispersive solid-phase extraction" for the determination of pesticide residues in produce. $J A O A C$ Int 86: 412-431

AOAC International (2007) AOAC Official Method 2007.01 Pesticide residues in foods by acetonitrile extraction and partitioning with magnesium sulfate. Official Methods of Analysis of AOAC International 90: 17-26. Retrieved from http://lib3.dss.go.th/fulltext/E_ content/1060-3271/2007v90n2.pdf
Breitenbach J, Changfu Z, Sandmann G. (2001) Bleaching herbicide norflurazon inhibits phytoene desaturase by competition with the cofactors. I Agric Food Chem 49: 5270-5272. https://doi. org/10.1021/JF0106751

Burgess SJ, Granero-Moya I, Grangé-Guermente MJ, Boursnell C, Terry MJ, Hibberd JM (2016) Ancestral light and chloroplast regulation form the foundations for C 4 gene expression. Nature Plants 2: 16161. https://doi.org/10.1038/nplants.2016.161

EN 15662:2008, Foods of plant origin - Determination of pesticide residues using GC-MS and/or LC-MS/MS following acetonitrile extraction / partitioning and cleanup by dispersive SPE - QuEChERS-method, European Committee for Standardization, 2008FAO/1/2017 Crop Prospects and Food Situation. Retrieved from http://www.fao.org/3/a-i6903e.pdf

Gil García MD, Uclés Duque S, Lozan o Fernández AB, Sosa A, Fernández-Alba AR (2017) Multiresidue method for trace pesticide analysis in honeybee wax comb by GC-QqQ-MS. Talanta 163: 54 64. https://doi.org/10.1016/j.talanta.2016.10.083

Holden M (1965) Analythical methods - Chlorophylls. In Chemistry and Biochemistry of Plant Pigments, Goodwin TW ed, p 463. London: Academic Press

Kümmel HW, Grimme LH (1975) The Inhibition of carotenoid biosynthesis in green algae by SANDOZ H 6706: accumulation of phytoene and phytofluene in Chlorella fusca. Z Naturforsch C 30: 333336. https://doi.org/10.1515/znc-1975-5-605

Moulin M, McCormac AC, Terry MJ, Smith AG (2008) Tetrapyrrole profiling in Arabidopsis seedlings reveals that retrograde plastid nuclear signaling is not due to Mg-protoporphyrin IX accumulation. Proc Natl Acad Sci USA 105: 15178-15183. https://doi.org/10.1073/ PNAS.0803054105

Neue UD (1997) HPLC Columns: Theory, Technology, and Practice. Wiley$\mathrm{VCH}$

Oelmüller R, Levitan I, Bergfeld R, Rajasekhar VK, Mohr H (1986) Expression of nuclear genes as affected by treatments acting on the plastids. Planta 168: 482-492. https://doi.org/10.1007/BF00392267

Pang GF, Fan CL, Liu YM, Cao YZ, Zhang JJ, Fu BL, Wu YP (2006) Multi-residue method for the determination of 450 pesticide residues in honey, fruit juice and wine by double-cartridge solid-phase extraction/gas chromatography-mass spectrometry and liquid chromatography-tandem mass spectrometry. Food Additives and Contaminants 23: 777-810. https://doi.org/10.1080/02652030600657997

Sandmann G, Linden H, Böger P (1989) Enzyme-kinetic studies on the interaction of norflurazon with phytoene desaturase. $Z$ Naturforsch C 44: 787-790. https://doi.org/10.1515/ZNC-1989-9-1015

SANTE/11945/2015 Guidance document on analytical quality control and method validation procedures for pesticides residues analysis in food and feed. European Comission Directorate-General for Health and Food Safety; 2015 (https://ec.europa.eu/food/sites/food/files/plant/docs/pesticides_ mrl_guidelines_wrkdoc_11945.pdf) 
Solymosi K, Aronsson H (2013) Etioplast and their significance in chloroplast biogenesis. In Plastid Development in Leaves During Growth and Senescence: Advances in Photosynthesis and Respiration, pp 39-71. https://doi.org/10.1007/978-94-007-5724-0

Strand $\AA$, Asami T, Alonso J, Ecker JR, Chory J (2003) Chloroplast to nucleus communication triggered by accumulation of $\mathrm{Mg}$-protoporphyrinIX. Nature 421: 79-83. https://doi.org/10.1038/nature01204

Terry MJ, Smith AG (2013) A model for tetrapyrrole synthesis as the primary mechanism for plastid-to-nucleus signaling during chloro- plast biogenesis. Front Plant Sci 4: 1-14. https://doi.org/10.3389/ fpls.2013.00014

Van Aken O, De Clercq I, Ivanova A, Law SR, Van Breusegem F, Millar AH, Whelan J (2016) Mitochondrial and chloroplast stress responses are modulated in distinct touch and chemical inhibition phases in Arabidopsis. Plant Physiol 171: 00273.2016. https://doi. org/10.1104/pp.16.00273 